\title{
Sleep apnea - another piece of the postoperative delirium puzzle?
}

\author{
Dennis Auckley
}

See related article by Roggenbach et al., http://ccforum.com/content/18/5/477

\begin{abstract}
Cardiac surgery is associated with high rates of postoperative delirium. The development of postoperative delirium increases morbidity, mortality and healthcare costs. Sleep disordered breathing is highly prevalent in patients undergoing cardiac surgery, and now appears likely to be an independent risk factor for postoperative delirium. If confirmed in larger controlled trials, sleep apnea may represent a risk factor for targeted intervention to reduce the rate of postoperative delirium.
\end{abstract}

\section{Introduction}

Postoperative delirium following cardiac surgery is extremely common and represents a challenging problem for perioperative care providers. It has been associated with increased length of hospital stay, postoperative morbidity (including prolonged cognitive dysfunction) and increased mortality. While multiple factors have been associated with delirium in the postoperative setting, evidence is mounting linking sleep apnea to this serious postoperative complication.

\section{Main text}

In this edition of Critical Care, Roggenbach and colleagues provide additional support to an association between obstructive sleep apnea (OSA) and postoperative delirium [1]. OSA has been linked to poor cardiovascular and neurologic outcomes, including cognitive dysfunction, in the outpatient setting [2,3]. While these studies have primarily focused on long-term outcomes, more immediate complications arising from OSA may be found in the perioperative setting where the added stress of surgery, anesthesia

Correspondence: dauckley@metrohealth.org

Division of Pulmonary, Critical Care and Sleep Medicine, MetroHealth Medical Center, 2500 MetroHealth Drive, Cleveland, OH 44109, USA and opioid use may magnify the effects of sleep disordered breathing.

There is now a wealth of data connecting OSA to significantly higher rates of postoperative cardiac and pulmonary complications [4]. However, the association between OSA and neurologic outcomes following surgery has received relatively little attention. Two earlier studies hinted at higher rates of postoperative delirium and encephalopathy in patients with OSA following hip and knee replacement and cardiac surgery, respectively, although these studies were limited by retrospective designs and nonstandardized definitions for the neurologic diagnoses $[5,6]$. A more recent prospective study found that older patients with polysomnogram-proven OSA undergoing hip and knee surgery more frequently experienced postoperative delirium than those without a known diagnosis of OSA ( $53 \%$ vs $20 \%, P=0.01$ ) [7]. This study added methodological strengths by excluding those with pre-existing cognitive dysfunction and utilizing standardized criteria for diagnosing postoperative delirium. However, by relying on a prior known diagnosis of OSA, the study probably underestimated the true prevalence of OSA in their population and this may have had an effect on the association.

Roggenbach and colleagues add to this growing body of literature by studying a cohort of patients undergoing elective cardiac surgery and ascertaining sleep disordered breathing severity just prior to surgery to maximize the chances of an accurate association between exposure and outcome. Utilizing a portable limited-channel sleep monitoring system, they found that $59 \%$ of the study population had at least moderate to severe sleep apnea (apnea-hypopnea index (AHI) $\geq 15$, and that the presence of preoperative sleep disordered breathing was associated with the development of delirium in the first 48 hours following surgery [1]. A preoperative AHI of 19 or higher correlated with a sixfold increased risk of postoperative delirium, and, after multivariate logistic regression analysis, the preoperative AHI, age, smoking and intraoperative blood transfusion remained the significant factors associated with 
postoperative delirium. Additional strengths of the study include a high enrollment rate, a standardized approach to anesthesia/postoperative sedation, and the lack of potentially confounding preoperative sleep apnea treatment (while continuous positive airway pressure was used postoperatively during the daytime for pulmonary issues, it was not used for the treatment of sleep apnea during sleep).

Of note, the authors chose to use a portable sleep study for the diagnosis of sleep apnea, and the findings in the study highlight the benefits as well as the limitations of this emerging technology when compared with the polysomnogram for the diagnosis of sleep apnea. Portable sleep monitoring is relatively inexpensive and convenient but the lack of technical oversight during the study can lead to inadequate data, as was found in 19\% of the recordings in this study where insufficient data resulted in the exclusion of 22 patients [1]. In addition, the inability of portable systems to monitor sleep often leads to an underestimation of the true AHI, which could affect the nature of the relationship between sleep apnea and postoperative delirium. Finally, as the authors acknowledge, the device used in this study did not measure respiratory effort, resulting in an inability to differentiate obstructive versus central apneas. This issue may also impact the association of sleep apnea and delirium, as these different types of sleep disordered breathing syndromes do not share the same etiologies and pathophysiologic consequences.

How OSA may contribute to the development of postoperative delirium remains a matter of speculation. OSA is frequently accompanied by nocturnal hypoxia, although measures of hypoxia by and large did not correlate with delirium in this study, as was also noted in the prior prospective study [7]. Another possible explanation may be that OSA contributes to subclinical vascular disease and this may become manifest under the stress of the postoperative environment. The proinflammatory state, seen chronically with OSA, may also be augmented by the extracorporeal circulation utilized during cardiac surgery and thus contribute to the development of delirium. Further investigation to better understand these mechanisms is warranted.

With this study, mounting data suggest that the diagnosis of sleep apnea is a risk factor for postoperative delirium. Many of the risk factors presently known to increase postoperative delirium, such as age, gender and co-morbidities, are not modifiable prior to surgery. OSA is a potentially modifiable risk factor amenable to preoperative intervention. However, before widespread screening and initiation of therapy for sleep apnea preoperatively can be recommended, evidence showing that interventions will impact outcomes is required. Limited data to date suggest there may be a role for continuous positive airway pressure therapy in reducing postoperative delirium in patients with OSA [5,7], although well-designed prospective controlled trials are needed.

\section{Conclusions}

Emerging data support an association between sleep disordered breathing, particularly OSA, and the development of postoperative delirium. If confirmed in larger controlled trials, OSA may represent a modifiable risk factor to reduce the rate of postoperative delirium, which could lead to improved patient outcomes and lower healthcare costs. Funding of large-scale clinical trials appears justified.

\section{Abbreviations}

AHI: Apnea-hypopnea index; OSA: Obstructive sleep apnea.

\section{Competing interests}

The author declares that he has no competing interests.

\section{Published online: 11 December 2014}

\section{References}

1. Roggenbach J, Klamann M, von Haken R, Bruckner T, Karck M, Hofer S: Sleep-disordered breathing is a risk factor for delirium after cardiac surgery: a prospective cohort study. Crit Care 2014, 18:477.

2. Golbin JM, Somers VK, Caples SM: Obstructive sleep apnea, cardiovascular disease, and pulmonary hypertension. Proc Am Thorac Soc 2008, 5:200-206.

3. Yaffe K, Laffan AM, Litwack Harrison S, Redline S, Spira AP, Ensrud KE, Ancoli-srael S, Stone KL: Sleep-disordered breathing, hypoxia, and risk of mild cognitive impairment and dementia in older women. JAMA 2011, 306:613-619.

4. Kaw R, Chung F, Pasupuleti V, Mehta J, Gay PC, Hernandez AV: Meta-analysis of the association between obstructive sleep apnoea and postoperative outcome. Br J Anaesth 2012, 109:897-906.

5. Gupta R, Parvizi J, Hanssen AD, Gay PC: Postoperative complications in patients with obstructive sleep apnea syndrome undergoing hip or knee replacement: a case-control study. Mayo Clin Proc 2001, 76:897-905.

6. Kaw R, Golish J, Ghamande S, Burgess R, Foldvary N, Walker E: Incremental risk of obstructive sleep apnea in cardiac surgical outcomes. I Cardiovasc Surg (Torino) 2006, 47:683-689.

7. Flink BJ, Rivelli SK, Cox EA, White WD, Falcone G, Vall TP, Young CC, Bolognesi MP, Krystal AD, Trzepacz PT, Moon RE, Kwatra MM: Obstructive sleep apnea and incidence of postoperative delirium after elective knee replacement in the nondemented elderly. Anesthesiology 2012, 116:788-796.

doi:10.1186/s13054-014-0667-x

Cite this article as: Auckley: Sleep apnea - another piece of the postoperative delirium puzzle? Critical Care 2014 18:667. 